\title{
Quintic quasi-topological gravity
}

\author{
Adolfo Cisterna, ${ }^{a, b}$ Luis Guajardo, ${ }^{c}$ Mokhtar Hassaïne ${ }^{c}$ and Julio Oliva ${ }^{d}$ \\ ${ }^{a}$ Vicerrectoría académica, Universidad Central de Chile, \\ Toesca 1783 Santiago, Chile \\ ${ }^{b}$ Instituto de Ciencias Físicas y Matemáticas, Universidad Austral de Chile, \\ Casilla 567, Valdivia, Chile \\ ${ }^{c}$ Instituto de Matemática y Física, Universidad de Talca, \\ Casilla 747, Talca, Chile \\ ${ }^{d}$ Departamento de Fúsica, Universidad de Concepción, \\ Casilla, 160-C, Concepción, Chile \\ E-mail: adolfo.cisterna@ucentral.cl, luis.guajardo.r@gmail.com, \\ hassaine@inst-mat.utalca.cl, julioolivazapata@gmail.com
}

ABSTRACT: We construct a quintic quasi-topological gravity in five dimensions, i.e. a theory with a Lagrangian containing $\mathcal{R}^{5}$ terms and whose field equations are of second order on spherically (hyperbolic or planar) symmetric spacetimes. These theories have recently received attention since when formulated on asymptotically AdS spacetimes might provide for gravity duals of a broad class of CFTs. For simplicity we focus on five dimensions. We show that this theory fulfils a Birkhoff's Theorem as it is the case in Lovelock gravity and therefore, for generic values of the couplings, there is no $s$-wave propagating mode. We prove that the spherically symmetric solution is determined by a quintic algebraic polynomial equation which resembles Wheeler's polynomial of Lovelock gravity. For the black hole solutions we compute the temperature, mass and entropy and show that the first law of black holes thermodynamics is fulfilled. Besides of being of fourth order in general, we show that the field equations, when linearized around AdS are of second order, and therefore the theory does not propagate ghosts around this background. Besides the class of theories originally introduced in arXiv:1003.4773, the general geometric structure of these Lagrangians remains an open problem.

KEYwords: Black Holes, Classical Theories of Gravity

ARXIV EPRINT: 1702.04676 


\section{Contents}

1 Introduction $\quad 1$

2 The theory 3

3 Static solutions $\quad 5$

4 Birkhoff's theorem $\quad 6$

$\begin{array}{lll}5 & \text { Black hole thermodynamics } & 7\end{array}$

6 No-ghosts on AdS 99

$\begin{array}{lll}7 & \text { Simple solutions for special values of the couplings } & 10\end{array}$

$\begin{array}{ll}7.1 \text { Quasi-topological gravity with a unique vacuum } & 10\end{array}$

$\begin{array}{lll}7.2 & \text { Pure quasi-topological gravity } & 11\end{array}$

8 Conclusions and final remarks $\quad 11$

$\begin{array}{ll}\text { A Coefficients of the quintic quasi-topological gravity } & 13\end{array}$

\section{Introduction}

The geometric classical description of the gravitational interaction naturally leads to General Relativity as the unique, diffeomorphism invariant theory with second order field equations in four dimensions. In spite of the successes the theory has had, the lack of a proper quantum description of gravitational phenomena led to the exploration of alternative scenarios which have received vast attention during the last decades. If one requires some level of predictability, such alternative scenarios might be restricted in some manner. As before, requirements such as diffeomorphism invariance and second order field equations are usually considered cornerstones in this process that naturally leads to Einstein-Hilbert action in three and four dimensions and to Lovelock theories in arbitrary dimension $D$ [1]. When formulated on asymptotically AdS spacetimes, using the tools of AdS/CFT correspondence [2] one can treat these theories as gravity duals of some Conformal Field Theory living at the boundary. As in the low energy limit of string theory, besides the EinsteinHilbert action for gravity, higher curvature corrections might appear in a perturbative treatment, which can differ in general from those in the Lovelock family. Nevertheless one can hope that some of the physics of these higher curvature corrections could be correctly captured by their Lovelock counterpart. These kind of explorations led for example to understand that finite contributions from quadratic terms might induce violations of the $\eta / s$ 
KSS bound [3] (see [4] and [5]). Lovelock theories provide one with a setup where certain control at a computational level can be attained. More precisely, in Lovelock theories one can find exact analytic static black holes which provide for finite temperature duals with interesting thermal properties and phase diagram structure. Within Lovelock family in five dimensions, General Relativity is supplemented by a single quadratic term (which is the dimensional continuation of the four-dimensional Euler density for Euclidean compact manifolds without boundary) and besides Newton's constant, the theory has an extra dimensionful coupling. In the context of the $\mathrm{AdS}_{5} / \mathrm{CFT}_{4}$ correspondence departing from this family would allow the dual CFT to be non-supersymmetric [6], at the cost of loosing some of the analytic computational control. A natural question therefore arises: is it possible to define a sensible gravity theory in five dimensions beyond the Einstein-Gauss-Bonnet theory? The so-called quasi-topological gravities provide for such an example. In reference [7] a new cubic gravity theory in five dimensions was introduced. The cubic combination reads

$$
\begin{aligned}
\mathcal{L}_{3}= & -\frac{7}{6} R_{c d}^{a b} R_{b f}^{c e} R^{d f}{ }_{a e}-R_{a b}{ }^{c d} R_{c d}{ }^{b e} R_{e}^{a}-\frac{1}{2} R_{a b}{ }^{c d} R_{c}^{a} R_{d}^{b} \\
& +\frac{1}{3} R_{b}^{a} R_{c}^{b} R_{a}^{c}-\frac{1}{2} R R_{b}^{a} R_{a}^{b}+\frac{1}{12} R^{3}
\end{aligned}
$$

and can be singled out as the unique cubic combination in five dimensions whose traced field equations lead to a second order constraint and that also has second order field equation on general spherically (planar or hyperbolic) symmetric spacetimes [8]. ${ }^{1}$ It was also realized in [7] that this theory belongs to a general family of Lagrangians of order $k$ in the curvature, that can be constructed in dimensions $D=2 k-1$ for $k \geq 3$, and have the simple form

$$
\begin{aligned}
\tilde{\mathcal{L}}_{k}= & \frac{1}{2^{k}}\left(\frac{1}{D-2 k+1}\right) \delta_{c_{1} d_{1} \cdots c_{k} d_{k}}^{a_{1} b_{1} \cdots a_{k} b_{k}}\left(C_{a_{1} b_{1}}^{c_{1} d_{1}} \cdots C_{a_{k} b_{k}}^{c_{k} d_{k}}-R_{a_{1} b_{1}}^{c_{1} d_{1}} \cdots R_{a_{k} b_{k}}^{c_{k} d_{k}}\right) \\
& -c_{k} C_{a_{1} b_{1}}^{a_{k} b_{k}} C_{a_{2} b_{2}}^{a_{1} b_{1}} \cdots C_{a_{k} b_{k}}^{a_{k-1} b_{k-1}} .
\end{aligned}
$$

Here $C_{a c d}^{b}$ is the Weyl tensor and

$$
c_{k}=\frac{(D-4) !}{(D-2 k+1) !} \frac{[k(k-2) D(D-3)+k(k+1)(D-3)+(D-2 k)(D-2 k-1)]}{\left[(D-3)^{k-1}(D-2)^{k-1}+2^{k-1}-2(3-D)^{k-1}\right]} .
$$

The Lagrangian (1.1) is obtained by setting $D=5$ and $k=3$ in (1.2) after expanding the Weyl tensor in terms of Riemann tensor and its traces. It's also interesting to note that after expanding the Lagrangian (1.2) in the case $k=2$, and then setting $D=3$, leads to the quadratic part of the New Massive Gravity Lagrangian [10].

The $\tilde{\mathcal{L}}_{k \geq 3}$ expression in (1.2) allows to directly verify that the linearized field equations around any conformally flat background ((A)dS in particular) are of second order. On the other hand, the relative factor $c_{k}$ in (1.2) is such that on spacetimes that are conformal to spherically (hyperbolic or planar) symmetric spacetimes, the higher derivative contributions to the field equations coming from the variations of the Weyl tensors, cancel each other. This is possible since for such family of spacetimes, all the component of the Weyl

\footnotetext{
${ }^{1}$ The quasi-topological combination found in [9] $\mathcal{Z}^{\prime}$, is related to $\mathcal{L}_{3}$ by the relation $\mathcal{L}_{3}=\frac{7}{24} \mathcal{Z}^{\prime}+\frac{7}{48} \mathcal{E}_{6}$, where $\mathcal{E}_{6}$ stands for the six-dimensional Euler that vanishes identically in five dimensions.
} 
tensor $C^{a b}{ }_{c d}$ are proportional to a single function $X$ [11], and therefore any scalar constructed as a complete contraction of $k$ Weyl tensors will be proportional to $X^{k}$ (see [12] and [13] for related further developments). These results allow to construct a new theory, quartic in the Riemann, in $D=7$.

Motivated by broadening the family of four-dimensional CFTs with relatively simple gravity duals, in reference [14] the authors successfully look for a quartic theory with second order field equations on spherical symmetry. Such theory was dubbed Quartic Quasi-topological gravity and when supplemented with terms of lower order in the Riemann tensor, leads to second order linearized field equations around AdS spacetime. A property that is shared by its cubic counterpart. It's important to notice that Quartic Quasi-topological gravity in five dimensions does not belong to the family defined in equation (1.2), nevertheless on spherically symmetric spacetimes, the field equations are simple and reduce to a generalized Wheeler-like polynomial equation for the lapse function [15] (see also [16]). The authors of [14] also conjecture the existence of quasi-topological gravities of arbitrarily high degree in the curvature. The purpose of the present paper is to show that for Lagrangians that are quintic in the curvature, $\mathcal{R}^{5}$, this is indeed the case.

The paper is organized as follows: in section II we show that there is at least one particular combination of quintic algebraic invariants in five dimension which leads to second order field equations on spherically symmetric spacetimes. In section III we present the quintic algebraic Wheeler's polynomial that determines the black hole solutions of the theory, we characterize the maximally symmetric solutions and show that for generic values of the couplings, the asymptotic behavior of the solutions coincides with the one of GR. Section IV is devoted to the proof of Birkhoff's theorem in this setup which shows that for generic values of the couplings of a non-homogeneous curvature combination, the theory does not propagate $s$-waves in spite of being in general a higher derivative theory. Due to the Abel-Rufini theorem the obtained Wheeler's-like polynomial cannot be solved by radicals, nevertheless, assuming the existence of an event horizon, we are able to compute the temperature, entropy and mass of the black hole solutions in section V. Section VI contains the proof that the theory is ghosts-free around AdS. In section VII we analyze some particular cases of the values of the couplings that allow for an explicit solution of the quintic polynomial and at the same time lead to interesting black hole solutions. Section VIII contains further remarks and conclusions.

\section{The theory}

Here we consider the following gravity theory

$$
I\left[g_{\mu \nu}\right]=\int \sqrt{-g} d^{5} x\left[\frac{R-2 \Lambda}{16 \pi G}+\sum_{k=2}^{5} a_{k} \mathcal{L}_{k}\right],
$$

where $\mathcal{L}_{2}$ stands for the Gauss-Bonnet combination

$$
\mathcal{L}_{2}:=R^{2}-4 R_{a b} R^{a b}+R_{a b c d} R^{a b c d},
$$


the cubic $\mathcal{L}_{3}$ term is the cubic quasi-topological combination in (1.1) and $\mathcal{L}_{4}$ stands for the quartic quasi-topological term that can be written as

$$
\begin{aligned}
\mathcal{L}_{4}= & \frac{1}{73 \times 2^{5} \times 3^{2}}\left[7080 R^{p q b s} R_{p b}^{a}{ }^{u} R_{a \text { u }}^{v{ }^{w}} R_{q v s w}-234 R^{p q b s} R_{p q}{ }^{a u} R_{a u}^{v w} R_{b s v w}-1237\left(R^{p q b s} R_{p q b s}\right)^{2}\right. \\
& +1216 R^{p q} R^{b s a u} R_{b s p}{ }^{v} R_{a u v q}-6912 R^{p q} R^{b s} R_{p q}^{a}{ }_{p}^{u} R_{a b u s}-7152 R^{p q} R^{b s} R_{p b}^{a u} R_{a u q s} \\
& +308 R^{p q} R_{p q} R^{b s a u} R_{b s a u}+298 R^{2} R^{p q b s} R_{p q b s}+12864 R^{p q} R^{b s} R_{b}^{a} R_{p s q a}-115 R^{4} \\
& \left.-912 R R^{p q} R^{b s} R_{p b q s}+4112 R^{p q} R_{p}^{b} R_{q}^{s} R_{b s}-4256 R R^{p q} R_{p}^{b} R_{q b}+1156 R^{2} R^{p q} R_{p q}\right] .
\end{aligned}
$$

The new quintic quasi-topological combination is

$$
\begin{aligned}
& \mathcal{L}_{5}=A_{1} R R_{b}^{a} R_{c}^{b} R_{d}^{c} R_{a}^{d}+A_{2} R R_{b}^{a} R_{a}^{b} R_{e f}^{c d} R_{c d}{ }^{e f}+A_{3} R R_{c}^{a} R_{d}^{b} R_{e f}^{c d} R_{a b}{ }^{e f} \\
& +A_{4} R_{b}{ }^{a} R_{a}^{b} R_{d}^{c} R_{e}^{d} R_{c}^{e}+A_{5} R_{b}^{a} R_{c}^{b} R_{a}^{c} R_{f g}^{d e} R_{d e}^{f g}+A_{6} R_{b}^{a} R_{d}^{b} R_{f}^{c} R_{a g}^{d e} R_{c e}{ }^{f g} \\
& +A_{7} R_{b}^{a} R_{d}^{b} R_{f}^{c} R_{c g}^{d e} R_{a e}^{f g}+A_{8} R_{b}^{a} R_{c}^{b} R_{a e}^{c d} R_{g h}{ }^{e f} R_{d f}^{g h}+A_{9} R_{b}^{a} R_{c}^{b} R_{e f}^{c d} R_{g h}{ }^{e f} R_{a d}^{g h} \\
& +A_{10} R_{b}^{a} R_{c}^{b} R_{e g}^{c d} R_{a h}^{e f} R_{d f}^{g h}+A_{11} R_{c}^{a} R_{d}^{b} R_{a b}{ }^{c d} R_{g h}{ }^{e f} R_{e f}^{g h}+A_{12} R_{c}^{a} R_{d}^{b} R_{a e}{ }^{c d} R_{g h}{ }^{e f} R_{b f}^{g h} \\
& +A_{13} R_{c}^{a} R_{d}^{b} R_{e f}^{c d} R_{g h}^{e f} R_{a b}{ }^{g h}+A_{14} R_{c}^{a} R_{d}^{b} R_{e g}^{c d} R_{a h}{ }^{e f} R_{b f}^{g h}+A_{15} R_{c}^{a} R_{e}^{b} R_{a f}^{c d} R_{g h}{ }^{e f} R_{b d}{ }^{g h} \\
& +A_{16} R_{b}{ }^{a} R_{a d}{ }^{b c} R_{f h}{ }^{d e} R_{c i}{ }^{f g} R_{e g}{ }^{h i}+A_{17} R_{b}{ }^{a} R_{d e}{ }^{b c} R_{c f}{ }^{d e} R_{h i}{ }^{f g} R_{a g}{ }^{h i}+A_{18} R_{b}{ }^{a} R_{d f}{ }^{b c} R_{a c}{ }^{d e} R_{h i}{ }^{f g} R_{e g}{ }^{h i} \\
& +A_{19} R_{b}{ }^{a} R_{d f}{ }^{b c} R_{a h}{ }^{d e} R_{e i}{ }^{f g} R_{c g}{ }^{h i}+A_{20} R_{b}{ }^{a} R_{d f}{ }^{b c} R_{g h}{ }^{d e} R_{e i}{ }^{f g} R_{a c}{ }^{h i}+A_{21} R_{c d}{ }^{a b} R_{e g}{ }^{c d} R_{a i}{ }^{e f} R_{f j}{ }^{g h} R_{b h}{ }^{i j} \\
& +A_{22} R_{c e}{ }^{a b} R_{a f}{ }^{c d} R_{g i}{ }^{e f} R_{b j}{ }^{g h} R_{d h}{ }^{i j}+A_{23} R_{c e}{ }^{a b} R_{a g}{ }^{c d} R_{b i}{ }^{e f} R_{f j}{ }^{g h} R_{d h}{ }^{i j}+A_{24} R_{c e}{ }^{a b} R_{f g}{ }^{c d} R_{h i}{ }^{e f} R_{a j}{ }^{g h} R_{b d}{ }^{i j},
\end{aligned}
$$

where the (not illuminating at all) $A_{k}$ coefficients, are defined in the appendix. The dimensionful couplings $a_{k}$ have mass dimension $5-2 k$.

The quasi-topological combinations seem to be very cumbersome but the field equations, on spherically symmetric spacetimes reduce to simple expressions.

Clearly, the quasi-topological gravities are defined up to the addition of the corresponding Euler densities that in the cubic, quartic and quintic cases, identically vanish in five dimensions. A different set of identities that can be used to provide a different expression for these Lagrangians make use of the cubic and quartic Lovelock tensor $\mathcal{G}_{\mu \nu}^{(3)}$ and $\mathcal{G}_{\mu \nu}^{(4)}$. These tensors identically vanish in dimension five and can be used to construct new scalar identities by contracting them with symmetric curvature combinations, as well as with curvature scalars. Beyond these trivial sources of non-uniqueness for the explicit form of the quasi-topological Lagrangians, there is a more subtle one that leads to theories that are intrinsically different. Since the quartic and quintic theories are defined by requiring second order field equations on spherically symmetric spacetimes, a combination that identically vanishes for this class of spacetimes can be added. The differences between the theories so defined will become manifest only when they are explored beyond spherical symmetry. An example of this is provided by the addition of a general combination of the independent, complete contractions of $k$ Weyl tensor $\operatorname{Tr}_{(p)}\left(C^{k}\right)$, where $\operatorname{Tr}_{(p)}$ stands for a particular way of pairing the indices of the different Weyl tensors. When evaluated on spacetimes that are conformal to spherically symmetric ones, the different traces $\operatorname{Tr}_{(p)}$ turn out to be proportional [11]. Therefore a combination of these traces, fixed by a single constraint, can be added to the quasi-topological terms to provide new theories which on spherical symmetry coincide (see the discussion above equation (88) in [7]). As mentioned in [8], since there are 
only two possible cubic complete contractions of Weyl tensors, $\operatorname{Tr}_{(1)}\left(C^{3}\right)$ and $\operatorname{Tr}_{(2)}\left(C^{3}\right)$, cubic quasi-topological gravity is unique. Quartic quasi-topological gravity is also argued to be unique in the original reference [14]. Whether or not the Quintic Quasi-topological theory here presented is unique, goes beyond the scope of this work and it would require at least to properly classify all the non-trivial, independent traces of the form $\operatorname{Tr}_{(p)}\left(C^{5}\right)$. In ten dimensions, these are the terms that define the $c$-contributions of the conformal anomaly (see e.g. [17] and [18]).

Hereafter we set $16 \pi G=1$.

\section{Static solutions}

Wheeler's polynomial for a static metric of the form

$$
d s^{2}=-f(r) d t^{2}+\frac{d r^{2}}{g(r)}+r^{2} d \Sigma_{\gamma}^{2}
$$

with $f(r)=g(r)$, reads

$$
(f-\gamma)^{5} \frac{a_{5}}{r^{6}}-(f-\gamma)^{4} \frac{a_{4}}{r^{4}}-2(f-\gamma)^{3} \frac{a_{3}}{r^{2}}-12(f-\gamma)^{2} a_{2}+6 r^{2}(f-\gamma)+r^{4} \Lambda=-\mu .
$$

Where $d \Sigma_{\gamma}$ denotes the line element of a Euclidean, three-dimensional manifold of normalized constant curvature $\gamma= \pm 1$ or 0 , and $\mu$ is an integration constant, that will determine the mass of the solution. This is a quintic algebraic equation that due to Abel-Rufini theorem, cannot be solved in general by radicals. We will show in the next section that for generic values of the couplings the condition $f(r)=g(r)$ is actually an output of the field equations and not a restriction put by hand.

Let's assume that the maximally symmetric solution has a dressed constant curvature

$$
R_{\beta \sigma}^{\mu \nu}=\frac{\lambda}{6}\left(\delta_{\beta}^{\mu} \delta_{\sigma}^{\nu}-\delta_{\sigma}^{\nu} \delta_{\beta}^{\mu}\right)
$$

whose metric can be represented by setting

$$
f(r)=g(r)=-\frac{\lambda}{6} r^{2}+\gamma .
$$

on the metric (3.1). The dressed curvature is fixed by the following polynomial

$$
P[\lambda]:=a_{5} \lambda^{5}+6 a_{4} \lambda^{4}-72 a_{3} \lambda^{3}+2592 a_{2} \lambda^{2}+7776(\lambda-\Lambda)=0 .
$$

As usual, depending on the values of the couplings $\left(a_{5}, a_{4}, a_{3}, a_{2}, \Lambda\right)$ one can have from one to five different vacua, and one can identify the G.R. branch, as the one for which $\lambda \rightarrow \Lambda$ as $a_{2}, a_{3}, a_{4}$ and $a_{5}$ go to zero.

It's possible to show that for generic values of the couplings, the asymptotic behavior allowed by Wheeler's polynomial (3.2), coincides with that of General Relativity. In fact assuming

$$
f(r)=g(r)=-\frac{\lambda}{6} r^{2}+\gamma+j(r)
$$


with $j(r)$ having a Laurent expansion at infinity that does not modify the leading term of the metric, i.e.

$$
j(r)=c_{1} r+c_{0}+\frac{c_{2}}{r}+\frac{c_{3}}{r^{2}}+\mathcal{O}\left(r^{-3}\right),
$$

one can expand (3.2) at infinity and solve it order by order. This leads to the following set of conditions

$$
P[\lambda] r^{4}=0, \quad c_{1} \frac{d P[\lambda]}{d \lambda} r^{3}=0,
$$

where $P[\lambda]$ is defined in (3.5). Once $P[\lambda]$ is solved, which fixes the value of the dressed cosmological constant $\lambda$, requiring at the same time $\frac{d P[\lambda]}{d \lambda}$ to vanish, would imply a relation between the couplings, therefore for generic values of the latter, (3.8) imply that $c_{1}$ has to vanish. Considering this on the next relevant order of the asymptotic expansion of Wheeler's polynomial one obtains

$$
c_{0} \frac{d P[\lambda]}{d \lambda} r^{2}=0
$$

which again implies that generically $c_{0}=0$. Then one obtains $c_{2} \frac{d P[\lambda]}{d \lambda} r=0$, which forces to set $c_{2}=0$, and finally one obtains

$$
c_{3} \frac{d P[\lambda]}{d \lambda}+1296 \mu=0 .
$$

As mentioned before, this implies that the asymptotic behavior of the full solution matches the one of General Relativity since

$$
f(r)=g(r)=-\frac{\lambda}{6} r^{2}+\gamma-\frac{\mu}{1296 \frac{d P[\lambda]}{d \lambda}} \frac{1}{r^{2}}+\mathcal{O}\left(r^{-3}\right) .
$$

This shows that on spherically symmetric spacetimes, the only way of relaxing the asymptotic conditions of quasi-topological gravity (with respect to those of G.R.), would be to consider the critical cases in which the polynomial $P[\lambda]$ has roots with multiplicity larger than one. In section VI we consider some particular regions of the space of couplings that allow for relaxed asymptotic behavior.

\section{Birkhoff's theorem}

It's well known that the field equations of a gravitational theory on spherically symmetric spacetimes, are correctly reproduced from the variation of a reduced action $[19,20]$, obtained from the evaluation of the Lagrangian on the metric

$$
d s^{2}=-a(t, r) b^{2}(t, r) d t^{2}+2 f(t, r) b(t, r) d t d r+\frac{d r^{2}}{a(t, r)}+r^{2} d \Sigma_{\gamma}^{2},
$$

where $d \Sigma_{\gamma}$ denotes the line element of a Euclidean three-dimensional manifold of constant curvature $\gamma= \pm 1$ or 0 . Evaluating the action (2.1) on (4.1) one obtains a reduced action which is a functional of $I[a, b, f]$. It's convenient to introduce $h(t, r)$ such that

$$
a(t, r)=\gamma+h(t, r)
$$


The variation of the reduced action with respect to $a, b$ and $f$, and a posteriori gauge fixing $f=0$, respectively lead to the field equations

$$
\begin{aligned}
0= & \left(-24 r^{6} h(t, r) a_{2}-6 h(t, r)^{2} r^{4} a_{3}-4 h(t, r)^{3} r^{2} a_{4}+5 h(t, r)^{4} a_{5}+6 r^{8}\right) \frac{\partial b(t, r)}{\partial r} \\
0= & h(t, r)^{5} r^{-5} a_{5}-h(t, r)^{4} r^{-3} a_{4}-2 h(t, r)^{3} r^{-1} a_{3}-12 h(t, r)^{2} r a_{2} \\
& +r^{5} \Lambda+6 r^{3} h(t, r)+\mu(t) r \\
0= & \left(-24 r^{6} h(t, r) a_{2}-6 h(t, r)^{2} r^{4} a_{3}-4 h(t, r)^{3} r^{2} a_{4}+5 h(t, r)^{4} a_{5}+6 r^{8}\right) \frac{\partial h(t, r)}{\partial t}
\end{aligned}
$$

The function $f(t, r)$ appears as a Lagrange multiplier on the reduced action and provides one with the $(t, r)$ component of the field equations. Here $\mu(t)$ is an integration function. For generic values of the couplings, provided (4.4) is fulfilled, one can show that the prefactors of the derivatives in equations (4.3) and (4.5) are non-vanishing and therefore $b(t, r)=b(t)$ and $\frac{\partial h}{\partial t}=0$. The latter implies $\mu(t)=\mu$. As usual the function $b(t)$ can be absorbed by a time reparametrization. This proves the Birkhoff's theorem for the nonhomogenous combination, i.e., for generic values of the couplings, the spherically (planar or hyperbolic) symmetric solution is static and is determined by a quintic polynomial equation. These results are analogous to the Birkhoff's theorem in Lovelock gravity (see [21] and [22]). It would be interesting to explore whether the generalized Birkhoff's theorem of ChernSimons [23] and Lovelock theory [24-33] can be extended for the quasi-topological theories, for general horizon geometries.

\section{Black hole thermodynamics}

Assuming that there is a non-degenerate horizon, that is, assuming that the polynomial equation (3.2) has a solution $f(r)$ with a single zero located at $r=r_{h}$, we now analyze the thermodynamical properties of the solution through the Euclidean method, where the Euclidean time $\tau$ is imaginary, periodic of period $\beta$ and it is related to the temperature via $\beta=T^{-1}$. The Euclidean action $I_{\text {Euc }}$ is related with the free energy $F$ by

$$
I_{\mathrm{Euc}}=\beta F=\beta(M-T \mathcal{S}),
$$

where $M$ is the mass and $\mathcal{S}$ is the entropy. On the other hand, in order to display the boundary term $B$ that will ensure the finiteness of the Euclidean action, it is enough to consider the following class of Euclidean metric

$$
d s^{2}=N^{2}(r) f(r) d \tau^{2}+\frac{d r^{2}}{f(r)}+r^{2} d \Sigma_{\gamma}^{2}
$$

With the Euclidean time $\tau \in[0, \beta]$, the radial coordinate $r \in\left[r_{h}, \infty\left[\right.\right.$ and $\operatorname{Vol}\left(\Sigma_{\gamma}\right)$ standing for the volume of the three-dimensional Euclidean manifold $\Sigma_{\gamma}$, the reduced action principle reads

$$
I_{\mathrm{Euc}}=\beta \operatorname{Vol}\left(\Sigma_{\gamma}\right) \int N(r) \mathcal{H}(r) d r+B,
$$


where the reduced Hamiltonian is given by

$$
\begin{aligned}
\mathcal{H}= & \left(\frac{5(f-\gamma)^{4} a_{5}}{2 r^{6}}-\frac{2(f-\gamma)^{3} a_{4}}{r^{4}}-\frac{3(f-\gamma)^{2} a_{3}}{r^{2}}-12(f-\gamma) a_{2}+3 r^{2}\right) f^{\prime} \\
& -\frac{3(f-\gamma)^{5} a_{5}}{r^{7}}+\frac{2(f-\gamma)^{4} a_{4}}{r^{5}}+\frac{2(f-\gamma)^{3} a_{3}}{r^{3}}+6 r(f-\gamma)+2 \Lambda r^{3} .
\end{aligned}
$$

The boundary term is determined requiring that the reduced action (5.2) has an extremum, that is, $\delta I_{\text {Euc }}=0$ within the class of fields considered here [34]. This last condition implies that

$$
\delta B=-\beta \operatorname{Vol}\left(\Sigma_{\gamma}\right) N\left(\frac{5(f-\gamma)^{4} a_{5}}{2 r^{6}}-\frac{2(f-\gamma)^{3} a_{4}}{r^{4}}-\frac{3(f-\gamma)^{2} a_{3}}{r^{2}}-12(f-\gamma) a_{2}+3 r^{2}\right) \delta f,
$$

where the variation is taken between the horizon and infinity.

Varying the reduced action with respect to the fields $N$ and $f$ gives the following equations:

$$
\begin{aligned}
& \mathcal{H}(r)=0 \\
& \left(-\frac{5(f-\gamma)^{4} a_{5}}{2 r^{6}}+\frac{\left.2(f-\gamma)^{3}\right) a_{4}}{r^{4}}+\frac{3(f-\gamma)^{2} a_{3}}{r^{2}}+12(f-\gamma) a_{2}-3 r^{2}\right) N^{\prime}(r)=0
\end{aligned}
$$

From the first equation we recover (3.2). In the second equation, since the couplings $a_{2}, a_{3}, a_{4}, a_{5}$ are generic, one must have $N^{\prime}(r)=0$, and then, without loss of generality, we can set $N(r) \equiv 1$.

The temperature is fixed requiring regularity of the metric at the horizon yielding in this case to

$$
\left.\beta f^{\prime}(r)\right|_{r_{h}}=4 \pi
$$

and using (3.2) we have

$$
T=\frac{1}{2 \pi r_{h}}\left(\frac{-3 a_{5} \gamma^{5}-2 a_{4} \gamma^{4} r_{h}^{2}+2 a_{3} \gamma^{3} r_{h}^{4}+6 \gamma r_{h}^{8}-2 \Lambda r_{h}^{10}}{5 a_{5} \gamma^{4}+4 a_{4} \gamma^{3} r_{h}^{2}-6 a_{3} \gamma^{2} r_{h}^{4}+24 a_{2} \gamma r_{h}^{6}+6 r_{h}^{8}}\right) .
$$

Now we are in position to compute the boundary term $B=B(\infty)-B\left(r_{h}\right)$. At the horizon we have $f\left(r_{h}\right)=0$ and $\left.\delta f\right|_{r_{h}}=-f^{\prime}\left(r_{h}\right) \delta r_{h}$. In this situation the variation reads

$$
\left.\delta B\right|_{r_{h}}=4 \pi \operatorname{Vol}\left(\Sigma_{\gamma}\right)\left(\frac{5 \gamma^{4} a_{5}}{2 r_{h}^{6}}+\frac{2 \gamma^{3} a_{4}}{r_{h}^{4}}-\frac{3 \gamma^{2} a_{3}}{r_{h}^{2}}+12 \gamma a_{2}+3 r_{h}^{2}\right) \delta r_{h},
$$

which can be trivially integrated as

$$
B\left(r_{h}\right)=\operatorname{Vol}\left(\Sigma_{\gamma}\right)\left(-\frac{2 \pi \gamma^{4} a_{5}}{r_{h}^{5}}-\frac{8 \pi \gamma^{3} a_{4}}{3 r_{h}^{3}}+\frac{12 \pi \gamma^{2} a_{3}}{r_{h}^{2}}+48 \pi \gamma a_{2} r_{h}+4 \pi r_{h}^{3}\right) .
$$

On the other hand, we can use again (3.2) to see that the variation at infinity is simply

$$
\left.\delta B\right|_{\infty}=\frac{\beta \operatorname{Vol}\left(\Sigma_{\gamma}\right)}{2} \delta \mu,
$$


so that the contribution at the infinity is given by

$$
\begin{aligned}
B(\infty) & =\frac{\beta \operatorname{Vol}\left(\Sigma_{\gamma}\right)}{2} \mu \\
& =-\frac{\beta \operatorname{Vol}\left(\Sigma_{\gamma}\right)}{2}\left(-\frac{\gamma^{5} a_{5}}{r_{h}^{6}}-\frac{\gamma^{4} a_{4}}{r_{h}^{4}}+\frac{2 \gamma a_{3}}{r_{h}^{2}}-12 \gamma^{2} a_{2}-6 \gamma r_{h}^{2}+\Lambda r_{h}^{4}\right) .
\end{aligned}
$$

Finally, the comparison between the boundary term $B$ and (5.1), allows us to identify the entropy and the mass of the system as

$$
\begin{aligned}
& \mathcal{S}=\operatorname{Vol}\left(\Sigma_{\gamma}\right)\left(4 \pi r_{h}^{3}+48 \pi \gamma a_{2} r_{h}+\frac{12 \pi \gamma^{2} a_{3}}{r_{h}^{2}}-\frac{8 \pi \gamma^{3} a_{4}}{3 r_{h}^{3}}-\frac{2 \pi \gamma^{4} a_{5}}{r_{h}^{5}}\right) . \\
& M=\frac{\operatorname{Vol}\left(\Sigma_{\gamma}\right)}{2}\left(\frac{\gamma^{5} a_{5}}{r_{h}^{6}}+\frac{\gamma^{4} a_{4}}{r_{h}^{4}}-\frac{2 \gamma a_{3}}{r_{h}^{2}}+12 \gamma^{2} a_{2}+6 \gamma r_{h}^{2}-\Lambda r_{h}^{4}\right)
\end{aligned}
$$

It's now trivial to show that the first law $d M=T d S$ is fulfilled.

As a final comment, we want to stress that the expression for the mass (5.9) coincides with the one obtained through the quasilocal generalization of the ADT formalism [35-39] as presented in refs. [40, 41].

\section{$6 \quad$ No-ghosts on AdS}

Linearizing fourth-order gravity theories around maximally symmetric backgrounds in general leads to ghost degrees of freedom. Some exceptions that non-trivially avoid this problem are New Massive Gravity as well as quasi-topological gravities. The former exploits the fact that General Relativity does not propagate local bulk degrees of freedom in $2+1$ dimensions, and the linearized equations around flat space lead to the massive Fierz-Pauli equation [10]. The mechanism that exorcizes the ghost on AdS in quasi-topological gravities is different. It was shown in [9] and [14] that around maximally symmetric backgrounds, cubic and quartic quasi-topological gravities lead to the same propagator than G.R. with an effective Newton's constant that depends on the values of the couplings. Requiring the positivity of this effective gravity coupling provides an important restriction on the values that the couplings can take. Now we focus on the linearization of Quintic Quasi-topological gravity, given by the action (2.1), around a maximally symmetric solution with a dressed curvature $\lambda / 6$, restricted by the polynomial $P[\lambda]=0$ in (3.5).

Recently, the authors of [42] and [43] introduced a simple method for linearizing higherderivative theories around maximally symmetric backgrounds. Their method relies on the evaluation of the Lagrangian on a deformed curvature that depends on two auxiliary parameters. Considering derivatives of the effective action with respect to the mentioned parameters, one can obtain the linearized field equations. The potentially dangerous terms contain second derivatives of the linearized Einstein tensor as well as of the linearized Ricci scalar. Such terms will be present for generic combinations of the curvature invariants. Nevertheless, for Quintic Quasi-topological gravity (2.1) one has that the linearized equa- 
tions around a constant curvature background of dressed curvature $\lambda / 6$ fixed by (3.5), $\operatorname{read}^{2}$

$$
\frac{d P[\lambda]}{d \lambda} G_{\mu \nu}^{L}=0
$$

that implies

$$
\frac{1}{2}\left(1+\frac{2}{3} a_{2} \lambda-\frac{1}{36} a_{3} \lambda^{2}+\frac{1}{324} a_{4} \lambda^{3}+\frac{5}{7776} a_{5} \lambda^{4}\right) G_{\mu \nu}^{L}=0
$$

where the linearized Einstein tensor is defined by

$$
\begin{aligned}
G_{\mu \nu}^{L}= & \bar{\nabla}_{(\mu \mid} \bar{\nabla}_{\sigma} h_{\mid \nu)}^{\sigma}-\frac{1}{2} \bar{\square} h_{\mu \nu}-\frac{1}{2} \bar{\nabla}_{\mu} \bar{\nabla}_{\nu} h+5 \Lambda h_{\mu \nu}-\Lambda h \bar{g}_{\mu \nu} \\
& -\frac{1}{2} \bar{g}_{\mu \nu}\left(\bar{\nabla}^{\alpha} \bar{\nabla}^{\beta} h_{\alpha \beta}-\bar{\square} h-4 \Lambda h\right)-4 \Lambda h_{\mu \nu} .
\end{aligned}
$$

The first term in the parenthesis of (6.2) comes from the Einstein-Hilbert term. The positivity of this effective Newton's constant has to be used to restrict the values of the couplings.

Note also that if we linearize around a maximally symmetric background that has a curvature that is a root of $P[\lambda]=0$ with multiplicity greater or equal to 2 , the linearized field equations would identically vanish. This feature is also well known in Lovelock theories when vacua coincide (see e.g. [44]).

\section{Simple solutions for special values of the couplings}

\subsection{Quasi-topological gravity with a unique vacuum}

As in Lovelock theory, one can see that if the couplings are such that all the roots of the polynomial $P[\lambda]$ in (3.5) coincide, Wheeler's polynomial can be solved in a simple manner $[45,46]$. For quasi-topological gravity, such degenerate case is obtained by setting the coefficients as

$$
\Lambda=-\frac{6}{5 l^{2}}, a_{2}=l^{2}, a_{3}=-6 l^{4}, a_{4}=6 l^{6}, a_{5}=\frac{6}{5} l^{8},
$$

where $l$ is an arbitrary length scale. In this case the metric can be found explicitly and yields

$$
d s^{2}=-\left(\frac{r^{2}}{l^{2}}+\gamma-\mu r^{6 / 5}\right) d t^{2}+\frac{d r^{2}}{\frac{r^{2}}{l^{2}}+\gamma-\mu r^{6 / 5}}+r^{2} d \Sigma_{\gamma}^{2},
$$

where $\mu$ is an integration constant. This spacetime is asymptotically locally (A)dS, which can be seen from the asymptotic form of the Riemann tensor at infinity

$$
R_{\alpha \beta}^{\mu \nu}=-\frac{1}{l^{2}} \delta_{\alpha \beta}^{\mu \nu}+p_{\alpha \beta}^{\mu \nu} \mathcal{O}\left(r^{-4 / 5}\right)
$$

where $p_{\alpha \beta}^{\mu \nu}$ is a tensor that has constant entries on the coordinates used for the line element (7.2).

\footnotetext{
${ }^{2}$ Note that for simplicity, we have scaled the curvature of the background w.r.t. that of [42] as $\lambda \rightarrow \lambda / 6$.
} 
Note also that the solution (7.2) can be obtained as a kind of dimensional continuation of the black hole solution of Lovelock theory with a unique vacuum. Indeed in such case the lapse function reads

$$
f(r)=\frac{r^{2}}{l^{2}}+\gamma-\frac{\mu}{r^{\frac{D-2 k-1}{k}}},
$$

where $D$ is the spacetime dimension and $k$ is the maximum power in the curvature that appears in the Lagrangian. Lovelock gravity of order $k$ contributes to the field equations provided $k \leq\left[\frac{D-1}{2}\right]$, where [.] stands for the integer part. Nevertheless, continuing the solution (7.4) to $D=5$ and $k=5$, correctly reproduces the line element (7.2). For cubic quasi-topological gravity, this property was already pointed out in [7].

\subsection{Pure quasi-topological gravity}

Another simple explicit solution arises when considering the quintic term plus a cosmological term in the action. In analogy to what happens with Lovelock gravity, we call this case the pure quasi-topological case. In the context of Lovelock theory, this case has received attention since it's the simplest case that admits a unique constant curvature solution that also propagates a graviton [47-49]. The theory therefore reads

$$
I_{P Q G}=\int \sqrt{-g} d^{5} x\left[-2 \Lambda+a_{5} \mathcal{L}_{5}\right]
$$

and the black holes solution reduces to

$$
d s^{2}=-\left(\gamma-\left(\frac{\left(\Lambda r^{4}+\mu\right) r^{6}}{a_{5}}\right)^{1 / 5}\right) d t^{2}+\frac{d r^{2}}{\gamma-\left(\frac{\left(\Lambda r^{4}+\mu\right) r^{6}}{a_{5}}\right)^{1 / 5}}+r^{2} d \Sigma_{\gamma}^{2}
$$

It's interesting enough to note that even in the absence of an Einstein-Hilbert term, the asymptotic behavior of the lapse function emulates that of GR, since at infinity

$$
f(r)=-\left(\frac{\Lambda}{a_{5}}\right)^{1 / 5} r^{2}+\gamma-\frac{\tilde{\mu}}{r^{2}}+\mathcal{O}\left(r^{-6}\right)
$$

The limit $\Lambda \rightarrow 0$ can be taken in this solution which provides an asymptotically locally flat black hole only for the hyperbolic case $\gamma=-1$.

\section{Conclusions and final remarks}

We have presented a new quasi-topological theory in five dimensions, that is quintic in the Riemann tensor. The theory is ghost-free around AdS and the linearized equations around a constant curvature background reduce to the linearized Einstein equations with an effective Newton's constant. The theory is defined by five, dimensionful coupling constants, and the positivity of the effective Newton's constant of the fluctuations on AdS has to be imposed as a restriction on the space of couplings. The theory also admits black hole solutions which can be integrated exactly up to the solution of a quintic algebraic equation, that due to Abel-Rufini theorem, cannot be solved in terms of radicals. Requiring the existence 
of an event horizon, we have been able to compute the temperature, mass and entropy of the black hole solutions. These black holes provide for finite temperature duals of a CFT living at the boundary of AdS spacetime.

It would be interesting to construct $\mathcal{R}^{5}$ quasi-topological gravities in arbitrary dimensions. From what we have learned from the cubic [7] and quartic cases [14], it is natural to conjecture that this theory might exist for all dimensions lower than ten, and from dimension 11 it might reduce to Lovelock theory plus a combination of invariants whose contribution cancels when evaluated on spherically symmetric spacetimes.

Cubic and quartic quasi-topological gravities have been explored in many different directions. Holographic and unitarity studies including $\eta / s$ and central charges of the dual CFTs were done in [50-52]. The properties of holographic superconductors were explored in [53-55]. Exact solution containing matter fields were constructed in [56-60] including also scalars conformally coupled to the quasi-topological densities [61]. The thermodynamics in the extended phase space for these theories was studied in [62] and [63]. It would be interesting to see if the results found in those references is affected by the presence of the quintic quasi-topological term, or whether the results are generic for this family of theories.

As mentioned above, besides the clear structure provided by the family of theories introduced in [7], of order $k$ in dimension $D=2 k-1$, the general form of quasi-topological gravities of arbitrary high order in five dimensions, is not clear. For the quartic case for example, one might be tempted to consider combinations of the invariants:

$$
\begin{aligned}
& B_{1}=\delta_{b_{1} \ldots b_{8}}^{a_{1} \ldots a_{8}} C^{b_{1} b_{2}}{ }_{a_{1} a_{2}} \ldots C^{b_{7} b_{8}} a_{a_{8}}, \quad \quad B_{2}=\delta_{b_{1} \ldots b_{8}}^{a_{1} \ldots a_{8}} R_{a_{1} a_{2}}^{b_{1} b_{2}} C_{a_{3} a_{4}}^{b_{3} b_{4}} \ldots C_{a_{7} a_{8}}^{b_{7} b_{8}}, \\
& B_{3}=\delta_{b_{1} \ldots b_{8}}^{a_{1} \ldots a_{8}} R_{a_{1} a_{2}}^{b_{1} b_{2}} R_{a_{3} a_{4}}^{b_{3} b_{4}} C_{a_{5} a_{6}}^{b_{5} b_{6}} C_{a_{7} a_{8}}^{b_{7} b_{8}}, \quad B_{4}=\delta_{b_{1} \ldots b_{8}}^{a_{1} \ldots a_{8}} R_{a_{1} a_{2}}^{b_{1} b_{2}} R_{a_{3} a_{4}}^{b_{3} b_{4}} R_{a_{5} a_{6}}^{b_{5} b_{6}} C_{a_{7} a_{8}}^{b_{7} b_{8}} \\
& B_{5}=\delta_{b_{1} \ldots b_{8}}^{a_{1} \ldots a_{8}} R_{a_{1} a_{2}}^{b_{1} b_{2}} R_{a_{3} a_{4}}^{b_{3} b_{4}} R_{a_{5} a_{6}}^{b_{5} b_{6}} R_{a_{7} a_{8}}^{b_{7} b_{8}} \text {. }
\end{aligned}
$$

Substituting the Weyl tensor in terms of the Riemann tensor and its traces, one can see that the combination

$$
\mathcal{B}=\frac{1}{(D-5)} \sum_{i=1}^{5} \xi_{i} B_{i},
$$

is well behaved for a general metric in $D=5$ if and only if

$$
\begin{aligned}
& \xi_{3}=-6 \xi_{1}-3 \xi_{2}, \\
& \xi_{4}=26 \xi_{1}+15 \xi_{2}, \\
& \xi_{5}=-21 \xi_{1}-13 \xi_{2} .
\end{aligned}
$$

Then, in order to remove the higher derivative contributions to the field equations one would have to engineer additional terms that on spherical symmetry contribute as those in the combinations that appear in $\mathcal{B}$. Besides the independent terms $\operatorname{Tr}_{(p)}\left(C^{4}\right)$, one might also consider $R \operatorname{Tr}_{(1)}\left(C^{3}\right)$ and $R \operatorname{Tr}_{(2)}\left(C^{3}\right)$.

\section{Acknowledgments}

We thank Gaston Giribet, Octavio Fierro and Sourya Ray for enlightening discussions and collaboration on similar topics. This work is partially supported by FONDECYT grants 
1130423, 1141073 and 3150157 . A.C work is partially supported by project UCEN I+D2016, CIP2016. This project is also partially funded by Proyectos CONICYT-Research Council UK-RCUK-DPI20140053. A.C. and J. O. would like to thank the International Center for Theoretical Physics (ICTP), Trieste, Italy, where part of this work was carried out. J.O. thanks also the support of ICTP Associates Program.

\section{A Coefficients of the quintic quasi-topological gravity}

To find the Quintic Quasi-topological combination, we have considered a general linear combination all the 85, quintic, curvature invariants that appear in the expansion of the ten-dimensional Euler density reported in equation (7) of reference [64]. We evaluated such general combination on a spherically (hyperbolic or planar) symmetric spacetime and then we required the field equations coming from this reduced Lagrangian to be of second order. This provides a system of equations for the coefficients of the linear combination, which has the coefficients given below as a simple, possible solution. Since we are interested in the properties of the theory on a spherically symmetric background, it's enough to evaluate the Lagrangian on the metric ansatz (3.1) (or (4.1) to include time dependence) [19, 20]. The coefficients that define the new Quintic Quasi-topological gravity in equation (2.4) are:

$$
\begin{aligned}
& A_{1}=\frac{9497}{17767320}, \quad A_{2}=-\frac{759299}{71069280}, \quad A_{3}=\frac{124967}{5922440}, \quad A_{4}=\frac{759299}{23689760}, \\
& A_{5}=\frac{197761}{11844880}, \quad A_{6}=\frac{1362599}{23689760}, \quad A_{7}=-\frac{5006573}{11844880}, \quad A_{8}=-\frac{9290347}{71069280}, \\
& A_{9}=\frac{3400579}{11844880}, \quad A_{10}=-\frac{6726521}{11844880}, \quad A_{11}=\frac{363777}{23689760}, \quad A_{12}=\frac{6348187}{47379520}, \\
& A_{13}=-\frac{9487667}{71069280}, \quad A_{14}=-\frac{6454201}{8883660}, \quad A_{15}=-\frac{34697591}{142138560}, \quad A_{16}=-\frac{5643853}{71069280}, \\
& A_{17}=-\frac{29094011}{71069280}, \quad A_{18}=-\frac{48458099}{71069280}, \quad A_{19}=\frac{1547591}{740305}, \quad A_{20}=\frac{78763919}{71069280}, \\
& A_{21}=-\frac{10718341}{17767320}, \quad A_{22}=\frac{9629717}{17767320}, \quad A_{23}=\frac{1113473}{17767320}, \quad A_{24}=-\frac{16111757}{17767320} \text {. }
\end{aligned}
$$

Open Access. This article is distributed under the terms of the Creative Commons Attribution License (CC-BY 4.0), which permits any use, distribution and reproduction in any medium, provided the original author(s) and source are credited.

\section{References}

[1] D. Lovelock, The Einstein tensor and its generalizations, J. Math. Phys. 12 (1971) 498 [INSPIRE].

[2] J.M. Maldacena, The large- $N$ limit of superconformal field theories and supergravity, Int. J. Theor. Phys. 38 (1999) 1113 [hep-th/9711200] [INSPIRE].

[3] P. Kovtun, D.T. Son and A.O. Starinets, Viscosity in strongly interacting quantum field theories from black hole physics, Phys. Rev. Lett. 94 (2005) 111601 [hep-th/0405231] [INSPIRE]. 
[4] M. Brigante, H. Liu, R.C. Myers, S. Shenker and S. Yaida, Viscosity bound violation in higher derivative gravity, Phys. Rev. D 77 (2008) 126006 [arXiv:0712.0805] [INSPIRE].

[5] M. Brigante, H. Liu, R.C. Myers, S. Shenker and S. Yaida, The viscosity bound and causality violation, Phys. Rev. Lett. 100 (2008) 191601 [arXiv:0802.3318] [INSPIRE].

[6] D.M. Hofman and J. Maldacena, Conformal collider physics: energy and charge correlations, JHEP 05 (2008) 012 [arXiv:0803.1467] [INSPIRE].

[7] J. Oliva and S. Ray, A new cubic theory of gravity in five dimensions: black hole, Birkhoff's theorem and C-function, Class. Quant. Grav. 27 (2010) 225002 [arXiv:1003.4773] [INSPIRE].

[8] J. Oliva and S. Ray, Classification of six derivative Lagrangians of gravity and static spherically symmetric solutions, Phys. Rev. D 82 (2010) 124030 [arXiv:1004.0737] [INSPIRE].

[9] R.C. Myers and B. Robinson, Black holes in quasi-topological gravity, JHEP 08 (2010) 067 [arXiv: 1003.5357] [INSPIRE].

[10] E.A. Bergshoeff, O. Hohm and P.K. Townsend, Massive gravity in three dimensions, Phys. Rev. Lett. 102 (2009) 201301 [arXiv:0901.1766] [INSPIRE].

[11] S. Deser and A.V. Ryzhov, Curvature invariants of static spherically symmetric geometries, Class. Quant. Grav. 22 (2005) 3315 [gr-qc/0505039] [inSPIRE].

[12] J. Oliva and S. Ray, Birkhoff's theorem in higher derivative theories of gravity, Class. Quant. Grav. 28 (2011) 175007 [arXiv:1104.1205] [INSPIRE].

[13] J. Oliva and S. Ray, Birkhoff's theorem in higher derivative theories of gravity II, Phys. Rev. D 86 (2012) 084014 [arXiv:1201.5601] [inSPIRE].

[14] M.H. Dehghani, A. Bazrafshan, R.B. Mann, M.R. Mehdizadeh, M. Ghanaatian and M.H. Vahidinia, Black holes in quartic quasitopological gravity, Phys. Rev. D 85 (2012) 104009 [arXiv: 1109.4708] [INSPIRE].

[15] J.T. Wheeler, Symmetric solutions to the maximally Gauss-Bonnet extended Einstein equations, Nucl. Phys. B 273 (1986) 732 [InSPIRE].

[16] X.O. Camanho and J.D. Edelstein, A Lovelock black hole bestiary, Class. Quant. Grav. 30 (2013) 035009 [arXiv:1103.3669] [INSPIRE].

[17] S. Deser and A. Schwimmer, Geometric classification of conformal anomalies in arbitrary dimensions, Phys. Lett. B 309 (1993) 279 [hep-th/9302047] [INSPIRE].

[18] N. Boulanger, Algebraic classification of Weyl anomalies in arbitrary dimensions, Phys. Rev. Lett. 98 (2007) 261302 [arXiv:0706.0340] [INSPIRE].

[19] R.S. Palais, The principle of symmetric criticality, Commun. Math. Phys. 69 (1979) 19 [INSPIRE].

[20] S. Deser and J. Franklin, Schwarzschild and Birkhoff à la Weyl, Am. J. Phys. 73 (2005) 261 [gr-qc/0408067] [INSPIRE].

[21] R. Zegers, Birkhoff's theorem in Lovelock gravity, J. Math. Phys. 46 (2005) 072502 [gr-qc/0505016] [INSPIRE].

[22] S. Deser and J. Franklin, Birkhoff for Lovelock redux, Class. Quant. Grav. 22 (2005) L103 [gr-qc/0506014] [INSPIRE]. 
[23] J. Oliva, All the solutions of the form $M_{2} \times{ }_{W} \sum_{d-2}$ for Lovelock gravity in vacuum in the Chern-Simons case, J. Math. Phys. 54 (2013) 042501 [arXiv:1210.4123] [INSPIRE].

[24] G. Dotti, J. Oliva and R. Troncoso, Static solutions with nontrivial boundaries for the Einstein-Gauss-Bonnet theory in vacuum, Phys. Rev. D 82 (2010) 024002 [arXiv: 1004.5287] [INSPIRE].

[25] G. Dotti, J. Oliva and R. Troncoso, Vacuum solutions with nontrivial boundaries for the Einstein-Gauss-Bonnet theory, Int. J. Mod. Phys. A 24 (2009) 1690 [arXiv:0809.4378] [INSPIRE].

[26] G. Dotti, J. Oliva and R. Troncoso, Exact solutions for the Einstein-Gauss-Bonnet theory in five dimensions: black holes, wormholes and spacetime horns, Phys. Rev. D 76 (2007) 064038 [arXiv:0706.1830] [inSPIRE].

[27] G. Dotti and R.J. Gleiser, Obstructions on the horizon geometry from string theory corrections to Einstein gravity, Phys. Lett. B 627 (2005) 174 [hep-th/0508118] [INSPIRE].

[28] C. Bogdanos, C. Charmousis, B. Gouteraux and R. Zegers, Einstein-Gauss-Bonnet metrics: black holes, black strings and a staticity theorem, JHEP 10 (2009) 037 [arXiv:0906.4953] [INSPIRE].

[29] H. Maeda, Gauss-Bonnet black holes with non-constant curvature horizons, Phys. Rev. D 81 (2010) 124007 [arXiv: 1004.0917] [INSPIRE].

[30] N. Farhangkhah and M.H. Dehghani, Lovelock black holes with nonmaximally symmetric horizons, Phys. Rev. D 90 (2014) 044014 [arXiv:1409.1410] [INSPIRE].

[31] S. Ohashi and M. Nozawa, Lovelock black holes with a nonconstant curvature horizon, Phys. Rev. D 92 (2015) 064020 [arXiv: 1507.04496] [INSPIRE].

[32] N. Dadhich and J.M. Pons, Static pure Lovelock black hole solutions with horizon topology $S^{(n)} \times S^{(n)}$, JHEP 05 (2015) 067 [arXiv: 1503.00974] [INSPIRE].

[33] S. Ray, Birkhoff's theorem in Lovelock gravity for general base manifolds, Class. Quant. Grav. 32 (2015) 195022 [arXiv:1505.03830] [INSPIRE].

[34] T. Regge and C. Teitelboim, Role of surface integrals in the Hamiltonian formulation of general relativity, Annals Phys. 88 (1974) 286 [INSPIRE].

[35] L.F. Abbott and S. Deser, Stability of gravity with a cosmological constant, Nucl. Phys. B 195 (1982) 76 [inSPIRE].

[36] L.F. Abbott and S. Deser, Charge definition in non-Abelian gauge theories, Phys. Lett. B 116 (1982) 259 [INSPIRE].

[37] S. Deser and B. Tekin, Gravitational energy in quadratic curvature gravities, Phys. Rev. Lett. 89 (2002) 101101 [hep-th/0205318] [INSPIRE].

[38] S. Deser and B. Tekin, Energy in generic higher curvature gravity theories, Phys. Rev. D 67 (2003) 084009 [hep-th/0212292] [INSPIRE].

[39] C. Senturk, T.C. Sisman and B. Tekin, Energy and angular momentum in generic F(Riemann) theories, Phys. Rev. D 86 (2012) 124030 [arXiv:1209.2056] [InSPIRE].

[40] W. Kim, S. Kulkarni and S.-H. Yi, Quasilocal conserved charges in a covariant theory of gravity, Phys. Rev. Lett. 111 (2013) 081101 [Erratum ibid. 112 (2014) 079902] [arXiv:1306.2138] [INSPIRE]. 
[41] Y. Gim, W. Kim and S.-H. Yi, The first law of thermodynamics in Lifshitz black holes revisited, JHEP 07 (2014) 002 [arXiv: 1403.4704] [INSPIRE].

[42] P. Bueno and P.A. Cano, Einsteinian cubic gravity, Phys. Rev. D 94 (2016) 104005 [arXiv: 1607.06463] [INSPIRE].

[43] P. Bueno, P.A. Cano, V.S. Min and M.R. Visser, Aspects of general higher-order gravities, Phys. Rev. D 95 (2017) 044010 [arXiv:1610.08519] [InSPIRE].

[44] Z.-Y. Fan, B. Chen and H. Lü, Criticality in Einstein-Gauss-Bonnet gravity: gravity without graviton, Eur. Phys. J. C 76 (2016) 542 [arXiv: 1606.02728] [INSPIRE].

[45] J. Crisostomo, R. Troncoso and J. Zanelli, Black hole scan, Phys. Rev. D 62 (2000) 084013 [hep-th/0003271] [INSPIRE].

[46] R. Aros, R. Troncoso and J. Zanelli, Black holes with topologically nontrivial AdS asymptotics, Phys. Rev. D 63 (2001) 084015 [hep-th/0011097] [INSPIRE].

[47] R.-G. Cai and N. Ohta, Black holes in pure Lovelock gravities, Phys. Rev. D 74 (2006) 064001 [hep-th/0604088] [INSPIRE].

[48] N. Dadhich, R. Durka, N. Merino and O. Mišković, Dynamical structure of pure Lovelock gravity, Phys. Rev. D 93 (2016) 064009 [arXiv:1511.02541] [InSPIRE].

[49] P.K. Concha, R. Durka, C. Inostroza, N. Merino and E.K. Rodríguez, Pure Lovelock gravity and Chern-Simons theory, Phys. Rev. D 94 (2016) 024055 [arXiv:1603.09424] [InSPIRE].

[50] R.C. Myers, M.F. Paulos and A. Sinha, Holographic studies of quasi-topological gravity, JHEP 08 (2010) 035 [arXiv: 1004.2055] [INSPIRE].

[51] M.H. Dehghani and M.H. Vahidinia, Quartic quasi-topological gravity, black holes and holography, JHEP 10 (2013) 210 [arXiv: 1307.0330] [INSPIRE].

[52] T.C. Sisman, I. Gullu and B. Tekin, All unitary cubic curvature gravities in D dimensions, Class. Quant. Grav. 28 (2011) 195004 [arXiv:1103.2307] [INSPIRE].

[53] M. Siani, Holographic superconductors and higher curvature corrections, JHEP 12 (2010) 035 [arXiv: 1010.0700] [INSPIRE].

[54] X.-M. Kuang, W.-J. Li and Y. Ling, Holographic superconductors in quasi-topological gravity, JHEP 12 (2010) 069 [arXiv:1008.4066] [INSPIRE].

[55] X.-M. Kuang, W.-J. Li and Y. Ling, Holographic p-wave superconductors in quasi-topological gravity, Class. Quant. Grav. 29 (2012) 085015 [arXiv: 1106. 0784] [InSPIRE].

[56] W.G. Brenna, M.H. Dehghani and R.B. Mann, Quasi-topological Lifshitz black holes, Phys. Rev. D 84 (2011) 024012 [arXiv: 1101.3476] [INSPIRE].

[57] M.H. Dehghani and M.H. Vahidinia, Surface terms of quasitopological gravity and thermodynamics of charged rotating black branes, Phys. Rev. D 84 (2011) 084044 [arXiv: 1108.4235] [INSPIRE].

[58] W.G. Brenna and R.B. Mann, Quasi-topological Reissner-Nordström black holes, Phys. Rev. D 86 (2012) 064035 [arXiv:1206.4738] [INSPIRE].

[59] M.A. Ganjali, Hyperscaling-violating Lifshitz solutions in cubic gravity, Phys. Rev. D 93 (2016) 024002 [arXiv:1508.05614] [INSPIRE]. 
[60] M. Ghanaatian, A. Bazrafshan, S. Taghipoor and R. Tawoosi, Magnetic brane of cubic quasi-topological gravity in the presence of Maxwell and Born-Infeld electromagnetic field, arXiv: 1609.06571 [INSPIRE].

[61] M. Chernicoff, O. Fierro, G. Giribet and J. Oliva, Black holes in quasi-topological gravity and conformal couplings, JHEP 02 (2017) 010 [arXiv:1612.00389] [INSPIRE].

[62] A. Sheykhi, M.H. Dehghani and R. Dehghani, Horizon thermodynamics and gravitational field equations in quasi-topological gravity, Gen. Rel. Grav. 46 (2014) 1679 [arXiv: 1404.0260] [INSPIRE].

[63] R.A. Hennigar, W.G. Brenna and R.B. Mann, $P-v$ criticality in quasitopological gravity, JHEP 07 (2015) 077 [arXiv:1505.05517] [INSPIRE].

[64] C.C. Briggs, A general expression for the quintic Lovelock tensor, gr-qc/9607033 [INSPIRE]. 